\title{
Immune Thrombocytopenic Purpura in Patients with COVID-19
}

\author{
Sabine Revuz, Nathalie Vernier, Leilah Saadi, Julien Campagne, Sophie Poussing, François Maurier \\ Hôpitaux Privés de Metz, Metz, France
}

Doi: 10.12890/2020_001751 - European Journal of Case Reports in Internal Medicine - ๑ EFIM 2020

Received: 27/05/2020

Accepted: 02/06/2020

Published: 09/06/2020

How to cite this article: Revuz S, Vernier N, Saadi L, Campagne J, Poussing S, Maurier F. Immune thrombocytopenic purpura in patients with COVID-19. EJCRIM 2020;7: doi:10.12890/2020_001751.

Conflicts of Interests: The Authors declare that there are no competing interests.

This article is licensed under a Commons Attribution Non-Commercial 4.0 License

\section{ABSTRACT}

We described three COVID-19-infected patients with profound immune thrombocytopenia causing haemorrhagic mucocutaneous complications. We conclude that an immune mechanism was responsible as common causes were excluded. Since corticoids were considered harmful in the circumstances, the patients were successfully treated with intravenous immunoglobulins without later relapse.

\section{LEARNING POINTS}

- The severity of haemorrhagic syndrome is not correlated with the severity of COVID-19 infection.

- Thrombocytopenia in mild COVID-19 infection seems to have an autoimmune mechanism.

- Intravenous immunoglobulins $(1 \mathrm{~g} / \mathrm{kg})$ should be the first line of treatment.

\section{KEYWORDS}

COVID-19, SARS-CoV-2, thrombocytopenia, intravenous immunoglobulins

\section{INTRODUCTION}

The COVID-19 pandemic started in China and was first described as a common cold and later as severe pneumonia. Many biological abnormalities were identified, including hypoxemia, hyperinflammation with extremely elevated C-reactive protein (CRP) and D-dimer levels, cholestasis, haemophagocytosis, high creatinine levels, electrolyte imbalance, derangement of the renin-angiotensin-aldosterone system and cytopenia. Thrombocytopenia has been associated with severe COVID-19 disease. We described three patients with peripheral thrombocytopenia considered to have an autoimmune aetiology as common causes were excluded. The patients were successfully treated with intravenous immunoglobulins.

\section{CASE DESCRIPTIONS}

\section{Case 1}

A 57-year-old woman was admitted with petechiae on her legs and gingival mucosae. She had a medical history of psoriatic arthritis treated with methotrexate, folic acid and adalimumab. She had presented 9 days previously with an influenza-like syndrome with cough, dyspnoea, headache, body aches, diarrhoea, anosmia and dysgeusia. At admission, she had a fever $\left(38.5 \mathrm{C}^{\circ}\right)$, normal blood pressure (116/56 mmHg), heart rate of 65 beats per minute, tachypnoea (respiratory rate 23 breaths per minute) and normal oxygen saturation of $95 \%$ on ambient air. Blood tests revealed severe thrombocytopenia ( $<1 \mathrm{~g} / \mathrm{l}$, without aggregates) without any other cytopenia, lymphocytosis with basophilia, elevated CRP (48 mg/l), normal fibrinogen (3.2 g/I), prothrombin 90\%, hepatic cytolysis, and normal creatinine (80 $\mu$ mol/I). Two SARSCoV-2 nasopharyngeal swabs taken 1 week apart were negative on RT-PCR testing. However, computed tomography (CT) of the chest showed ground-glass opacities in the lower zones (15\% of lung parenchyma), strongly suggestive of COVID-19 infection. 
As an immune mechanism was considered likely, the patient received a single infusion of intravenous immunoglobulins ( $1 \mathrm{~g} / \mathrm{kg}$ of body weight). Her platelet count progressively normalized and was stable 1 month later. Immunosuppressive treatment was postponed for 4 weeks.

Case 2

A 76-year-old man was admitted for fever and slight purpura on his legs. He had a history of metastatic bronchiolar adenocarcinoma with a PDL-1 expression level of $100 \%$, treated with carboplatin, pemetrexed and pembrolizumab with a good response. Blood tests showed thrombocytopenia (17 g/l), lymphopenia ( $0.59 \mathrm{~g} / \mathrm{l})$, normocytic anaemia ( $8.8 \mathrm{~g} / \mathrm{dl})$, normal prothrombin and fibrinogen levels, no schistocytes, elevated CRP (67 mg/l), hyperferritinemia (3544 $\mu \mathrm{g} / \mathrm{l})$, hepatic cytolysis and cholestasis. A nasopharyngeal swab was positive for COVID-19 on RT-PCR testing. A bone marrow smear showed abundant cell lines and no haematophagocytosis. There were no anti-PF4heparin antibodies. Consumption coagulopathy and haemolysis were excluded. The patient was treated with ciprofloxacin, ceftazidime, and low-weight heparin which was stopped after COVID-19 identification. The most severe thrombocytopenia (13 g/l) occurred 11 days after the first symptoms of SARS-CoV-2 infection. To prevent haemorrhagic complications, the patient received an infusion of intravenous immunoglobulins ( $1 \mathrm{~g} / \mathrm{kg}$ of body weight) for 2 days. His platelet count returned to normal in 7 days.

\section{Case 3}

A 39-year-old man was admitted for fever $\left(>38.5^{\circ} \mathrm{C}\right)$, arthralgias, cough, body aches and unilateral epistaxis 7 days after exposure to his two sons who had RT-PCR-confirmed COVID-19. He had a medical history of immune thrombocytopenic purpura (ITP) in 2005, treated with Disulone, which had been stopped several years before admission. Blood tests revealed thrombocytopenia (38 g/l), lymphopenia (0.8 $\mathrm{g} / \mathrm{I}$ ) and a normal CRP. No sign of coagulopathy was found. His baseline platelet count was $50 \mathrm{~g} / \mathrm{l}$. A nasopharyngeal swab was positive for SARS-CoV-2 on RT-PCR testing. In light of the reduced platelet count, a single infusion of intravenous immunoglobulins ( $1 \mathrm{~g} / \mathrm{kg}$ of body weight) was given, significantly improving the platelet count to $88 \mathrm{~g} / \mathrm{l}$.

\section{DISCUSSION}

We have described three COVID-19-infected patients with haemorrhagic symptoms (petechiae, epistaxis) very likely caused by immune thrombocytopenia. Notably, potential risk factors were identified in the patients' medical history: a previous ITP episode, chemotherapy, immunotherapy and immunosuppressive drugs. The patients did not present with severe haemorrhage. Intravenous immunoglobulin infusion (1-2 g/kg of body weight) was effective and response seemed to be stable 1 month after the development of the first symptoms. No corticosteroid therapy was administered.

The authors of a study published in March, of patients in Wuhan (China), where the COVID-19 pandemic began, did not mention thrombocytopenia ${ }^{[1]}$. However, in a meta-analysis ${ }^{[2]}$, thrombocytopenia was associated with a severe form of COVID-19, as an adverse prognostic factor, similar to high CRP or D-dimer levels. In this meta-analysis, thrombocytopenia was not found to be severe (platelet count $>80 \mathrm{~g} / \mathrm{I})$ and may be part of the classic cytokine storm in infectious disease. The first case of ITP in a patient with COVID-19 infection has already been published and intravenous immunoglobulins were given ${ }^{[3]}$.

In our patients, classic causes of thrombocytopenia were first excluded: consumption coagulopathy, toxic shock, heparin-induced thrombopenia, haemophagocytic syndrome and thrombotic thrombocytopenic purpura. Patient background, prothrombin time, activated partial thromboplastin time, fibrinogen and D-dimer levels, schistocytes and bone marrow aspiration findings suggested the mechanism of thrombocytopenia (peripheral vs central, fibrinogen consumption or not) and thus identified immune thrombocytopenia. In addition, flares in primary immune thrombocytopenia are initiated by an infectious trigger ${ }^{[4]}$.

SARS-CoV-2 induces diffuse endothelial dysfunction causing diffuse microangiopathy. A hyperinflammatory syndrome and haemostasis perturbation lead to hypercoagulopathy and microvascular immunothrombosis with multiple occlusions and microthrombi, especially in the pulmonary vasculature. Thrombocytes are involved in local haemostasis and immunothrombosis leading to mild thrombocytopenia in severe cases of COVID-19 ${ }^{[5]}$.

\section{CONCLUSIONS}

SARS-CoV-2 infection can cause profound and symptomatic thrombocytopenia. However, the severity of haemorrhagic signs is not related to COVID-19 severity. Many mechanisms sustain peripheral thrombocytopenia in COVID-19. An immune aetiology should be considered when other mechanisms have been ruled out in profound and acute thrombocytopenia. SARS-CoV-2 provokes an immune reaction which has a good response to intravenous immunoglobulins in mild or moderate COVID-19. 


\section{REFERENCES}

1. Chen T, Wu D, Chen H, Yan W, Yang D, Chen G, et al. Clinical characteristics of 113 deceased patients with coronavirus disease 2019: retrospective study. BMJ 2020;368:m1091.

2. Lippi G, Plebani M, Henry BM. Thrombocytopenia is associated with severe coronavirus disease 2019 (COVID-19) infections: a meta-analysis. Clin Chim Acta 2020;506:145148.

3. Zulfiqar AA, Lorenzo-Villalba N, Hassler P, Andrès E. Immune thrombocytopenic purpura in a patient with Covid-19. N Engl J Med 2020;382(18):e43.

4. Provan D, Arnold DM, Bussel JB, Chong BH, Cooper N, Gernsheimer T, et al. Updated international consensus report on the investigation and management of primary immune thrombocytopenia. Blood Adv 2019;3:3780-3817.

5. Henry BM, Vikse J, Benoit S, Favaloro EJ, Lippi G. Hyperinflammation and derangement of renin-angiotensin-aldosterone system in COVID-19: a novel hypothesis for clinically suspected hypercoagulopathy and microvascular immunothrombosis. Clin Chim Acta 2020;507:167-173. 